\title{
UV-Vis Spectroscopic Characterization of Nanomaterials in Aqueous Media
}

Ana C. Quevedo ${ }^{1}$, Emily Guggenheim ${ }^{1}$, Sophie M. Briffa ${ }^{1}$, Jessica Adams ${ }^{2,3}$, Stephen Lofts ${ }^{2}$, Minjeong Kwak ${ }^{4}$, Tae Geol Lee $^{4}$, Colin Johnston ${ }^{5}$, Stephan Wagner $^{6}$, Timothy R. Holbrook ${ }^{6}$, Yves U. Hachenberger ${ }^{7}$, Jutta Tentschert ${ }^{7}$, Nicholas Davidson ${ }^{1}$, Eugenia Valsami-Jones ${ }^{1}$

${ }^{1}$ School of Geography, Earth and Environmental Sciences, University of Birmingham ${ }^{2}$ UK Centre for Ecology and Hydrology ${ }^{3}$ Natural England ${ }^{4}$ Center for Nanosafety Metrology, Korea Research Institute of Standards and Science (KRISS) ${ }^{5}$ Department of Materials, University of Oxford ${ }^{6}$ Department of Analytical Chemistry, Helmholtz-Centre for Environmental Research ${ }^{7}$ Department of Chemical and Product Safety, German Federal Institute for Risk Assessment (BfR)

\section{Corresponding Author}

Eugenia Valsami-Jones

E.ValsamiJones@bham.ac.uk

\section{Citation}

Quevedo, A.C., Guggenheim, E., Briffa, S.M., Adams, J., Lofts, S., Kwak, M., Lee, T.G., Johnston, C., Wagner, S., Holbrook, T.R.,

Hachenberger, Y.U., Tentschert, J., Davidson, N., Valsami-Jones, E. UVVis Spectroscopic Characterization of Nanomaterials in Aqueous Media. J. Vis. Exp. (176), e61764, doi:10.3791/61764 (2021).

\section{Date Published}

October 25, 2021

\section{DOI}

$10.3791 / 61764$

\section{URL}

jove.com/video/61764

\section{Abstract}

The physicochemical characterization of nanomaterials (NMs) is often an analytical challenge, due to their small size (at least one dimension in the nanoscale, i.e. 1-100 $\mathrm{nm}$ ), dynamic nature, and diverse properties. At the same time, reliable and repeatable characterization is paramount to ensure safety and quality in the manufacturing of NMbearing products. There are several methods available to monitor and achieve reliable measurement of nanoscale-related properties, one example of which is UltravioletVisible Spectroscopy (UV-Vis). This is a well-established, simple, and inexpensive technique that provides non-invasive and fast real-time screening evaluation of NM size, concentration, and aggregation state. Such features make UV-Vis an ideal methodology to assess the proficiency testing schemes (PTS) of a validated standard operating procedure (SOP) intended to evaluate the performance and reproducibility of a characterization method. In this paper, the PTS of six partner laboratories from the $\mathrm{H} 2020$ project ACEnano were assessed through an interlaboratory comparison (ILC). Standard gold (Au) colloid suspensions of different sizes (ranging 5-100 nm) were characterized by UV-Vis at the different institutions to develop an implementable and robust protocol for NM size characterization.

\section{Introduction}

Nanomaterials (NMs) have become popular due to their unique properties in the nanoscale (1 to $100 \mathrm{~nm}$ ), which differ from the properties of their bulk counterparts, either due to size-related or quantum effects (e.g., increased specific surface area by volume) along with distinct reactivity, optical, thermal, electrical, and magnetic properties ${ }^{1,2}$. The potential applications of NMs in society are diverse and widely related to fields such as health care, food industry, cosmetics, paints, 
coatings, and electronics ${ }^{3,4,5}$. Gold nanoparticles (AuNPs) are widely applied in nanotechnology (e.g., in health care, cosmetics, and electronic applications), mainly due to their simple fabrication, size-dependent optical features, surface functionalization potential, and physicochemical properties, which can be suitable for many key applications ${ }^{6,7}$.

Quality and reproducibility in the synthesis and characterization of NMs are extremely important for quality assurance, but also for the safe manufacture of nano-based products, especially due to the reactivity of NMs, notably in complex environments, where NM properties, such as size distribution and morphology, may undergo rapid changes 8,9 . Numerous methods are available to monitor nanoscalerelated properties. For example, scanning/transmission electron microscopy (SEM/TEM) are techniques used to obtain high-resolution (down to sub-nanometer) optical and compositional information of NMs; atomic force microscopy (AFM) provides nanoscale resolution in the vertical ( $z$ axis) dimension; and X-ray diffraction (XRD) provides information on the atomic structure of NMs; all these methods can only be used on dry samples (powders) ${ }^{10,11}$. Techniques suitable for the characterization of NMs in liquid media include field flow fractionation (FFF), which allows the separation of large molecules, aggregates, and particles based on their size; dynamic light scattering (DLS); and nanoparticle tracking analysis (NTA) - two methods widely used to determine the size distribution profile of particles using Brownian motion —and ultraviolet-visible spectrophotometry (UV-Vis), which allows the assessment of NM characteristics such as size, aggregation state, and refractive index by a simple absorption measurement ${ }^{11,12,13}$. Although all these techniques allow NM characterization, their performance is dependent on instrument setup, instrument-related differences, complex methodology for sample preparation, and the user's level of expertise. Moreover, most of the techniques do not allow real-time monitoring of NM size, sample integrity, or differentiation between dispersed or aggregated particles ${ }^{6}$. UV-Vis spectroscopy is a widely used technique that provides non-invasive and fast real-time evaluation of NM size, concentration, and aggregation state. Additionally, it is a simple and inexpensive process with minimal sample preparation, which makes this technique an essential tool that is extensively used in numerous laboratories within many disciplines and markets ${ }^{6,12,14}$. UV-Vis works by measuring the transmittance of electromagnetic radiation of a wavelength between 180 and $1100 \mathrm{~nm}$ through a liquid sample. The UV and VIS spectral ranges cover the wavelength range for the ultraviolet $(170 \mathrm{~nm}$ to $380 \mathrm{~nm})$, visible ( $380 \mathrm{~nm}$ to $780 \mathrm{~nm}$ ), and near-infrared $(780 \mathrm{~nm}$ to 3300 $\mathrm{nm})^{4,14}$. The wavelength of light passing through the sample cell is measured; the intensity of light entering the sample is referred to as $\mathrm{I}_{0}$, and the intensity of the light emerging on the other side is designated as $\mathrm{I}_{1}{ }^{14}$. The Beer-Lambert law reflects the relationship between $A$ (absorbance) as a function of sample concentration C, the sample extinction coefficient $\epsilon$, and the two intensities ${ }^{14}$. Absorption measurements can be collected at a single wavelength or over an extended spectral range; the measured light transmittance is transformed into an absorbance measurement by following the Beer-Lambert law equation. The standard equation for absorbance is $A$ $=$ Elc, where $(A)$ is the amount of light absorbed by the sample for a given wavelength $(\varepsilon)$ is the molar attenuation coefficient (absorbance/(g/dm ${ }^{3}$ ) (l) is the distance the light travels through the solution $(\mathrm{cm})$, and $(\mathrm{c})$ is the concentration per unit volume $\left(\mathrm{g} / \mathrm{dm}^{3}\right)$. The absorbance is calculated as the ratio between the intensity of a reference sample $\left(\mathrm{I}_{0}\right)$ and the unknown sample (I), as described in the following equation ${ }^{14}$ : 
$A=\log 10\left(\frac{I_{0}}{I}\right)=\varepsilon l C$

The simplicity of UV-Vis makes it an ideal technique to compare PTS of an established measurement protocol $^{6,12,15}$. The objective of an ILC or PTS is to verify the performance and reproducibility of a method using an SOP $^{15}$. This, in turn, provides a standardized approach for quick characterization of nanoparticle suspensions for other users.

To assess the proficiency, consistency, and reliability of the method presented here, six laboratories participated in an ILC as members of the Horizon 2020 ACEnano project (https://cordis.europa.eu/project/id/720952). The ILC involved UV-Vis characterization of standard Au colloid dispersions of different particle sizes (5-100 nm). An SOP was provided to all the involved laboratories to ensure the identical preparation of AuNP suspensions, evaluation, and reporting of results to contribute towards the development of an implementable and robust tiered approach in NM physicochemical characterization, data interpretation, and improvement of best practice protocols for industrial and regulatory needs ${ }^{8}$.

\section{Protocol}

\section{Delivery of the AuNP samples:}

1. Prepare aliquots of $5 \mathrm{~mL}$ of Au colloid dispersions with sizes of $5,20,40,60$, and $100 \mathrm{~nm}$ including a $50 \mu \mathrm{g} /$ $\mathrm{mL}$ sample of 'unknown size' (See Table of Materials for more specific details about the nanomaterials used).

2. Send the samples in $7 \mathrm{~mL}$ polystyrene containers with gel packs to each participating laboratory to maintain a suitable temperature during the shipping. Store the samples at $4{ }^{\circ} \mathrm{C}$ immediately.

NOTE: The 'unknown size' sample must present a size of $80 \mathrm{~nm}$; this information should be known by the partner distributing the material, but not disclosed to the other partners.

\section{Calibration of the spectrophotometer:}

1. Turn on the UV-Vis spectrometer for at least $20 \mathrm{~min}$ to allow the lamp to heat up.

NOTE: Refer to the Table of Materials for the model and brand of the spectrophotometer used.

2. In the software, select the option Spectrum scan from the mode window, which displays the operating modes.

3. Adjust the parameter settings in Instrument | Settings and parameters in the software before proceeding with measurements: Measurement Mode | Spectrum scan, Data Mode | ABS, Start wavelength of $680 \mathrm{~nm}$, End Wavelength of $380 \mathrm{~nm}$, Scan Speed of $400 \mathrm{~nm} / \mathrm{min}$, Sampling interval of 0.5 , Slit Width of 1.5 , and Path Length of 10.

4. After the parameters have been set, fill two cuvettes (3 $\mathrm{mL}$; polystyrene) with $1 \mathrm{~mL}$ of ultrapure water (UPW) $(18.2 \mathrm{M} \cdot \Omega \cdot \mathrm{cm})$. Place the cuvettes in the reference cell holder (rear) and the sample cell holder (front) to cover the light path (See Table of Materials for the specific brand and model of the cuvettes used).

NOTE: Make sure the cuvettes are positioned and aligned correctly to cancel the noise effect and other environmental effects that are not sample-related.

5. Close the UV-Vis instrument cover and continue with the blank calibration by selecting Blank from the command bar. The baseline correction is performed by running a 
reference with the two cuvettes filled with $1 \mathrm{~mL}$ of UPW placed in the sample holders. For alternative protocols used by other partners, please see Supplementary Information (SI).

\section{Preparation of the samples}

1. Take a subsample of $500 \mu \mathrm{L}$ for each AuNP of $5,20,40$, $60,100 \mathrm{~nm}$, and the unknown size, and prepare a dilution with $500 \mu \mathrm{L}$ of UPW.

2. Place the diluted suspensions in $1 \mathrm{~mL}$ cuvettes; the total dilution ratio should be 1:1 and final concentration $25 \mu \mathrm{g} /$ $\mathrm{mL}$.

NOTE: The diluted sample must be prepared immediately before the UV-Vis measurement.

\section{Measurement of the nanoparticle dispersions}

1. After the blank calibration has been performed, and a fresh sample has been prepared, replace one of the blank cuvettes in the sample cell holder (front) with the AuNP dispersion sample; the other reference cuvette filled with $1 \mathrm{~mL}$ of UPW must be left untouched.

NOTE: Use a new disposable cuvette for different samples to avoid cross-contamination between samples. When using quartz cuvettes, rinse the sample cuvette with UPW between samples.

2. Select the option Measure/Start from the command bar to run the spectrum scans for each diluted AuNP dispersion. Three spectrum scan runs should be obtained for each AuNP sample, including the unknown size sample.

NOTE: Ensure that the blank cuvette remains in the reference cell holder when running a measurement.

\section{Reporting results}

1. Extract the raw experimental data for each measurement in a spreadsheet-compatible file by selecting File menu and clicking Export report (*.csv) file.

2. Note the maximum absorption wavelength (Absmax) and lambda $\left(\lambda_{\max }\right)$ for each of the UV-Vis readings and record them in the provided template.

NOTE: The predesigned template was provided to the ACEnano partners to automatically calculate the wavelengths' average standard deviations by setting the appropriate calculation formula in the workbook. For further details and access to the template, see Supplementary Information (SI).

3. In the workbook, plot a calibration curve with the average of the $\lambda_{\max }(y$-axis) against the known nanoparticle size (nm) $(5,20,40,60$, and $100 \mathrm{~nm})$. For example, in the spreadsheet, create the calibration curve by selecting in the command bar Data | Insert Graph | Scatter Plot | Add Trendline | Polynomial Curve (Power 2).

4. Include the polynomial equation for the calibration curve: select Trendline options | Display Equation On Chart from the command bar (Figure 1).

5. Finally, to calculate the unknown size of the AuNP sample, isolate the polynomial equation from the calibration curve to fit the mean value for the unknown $\lambda_{\max }$, using a derivation of the quadratic formula (Figure 1). The calculated size can be included in the template to complete a full summary of the data for consistency, faster interpretation, and evaluation of the results (see $\mathrm{SI})$. 


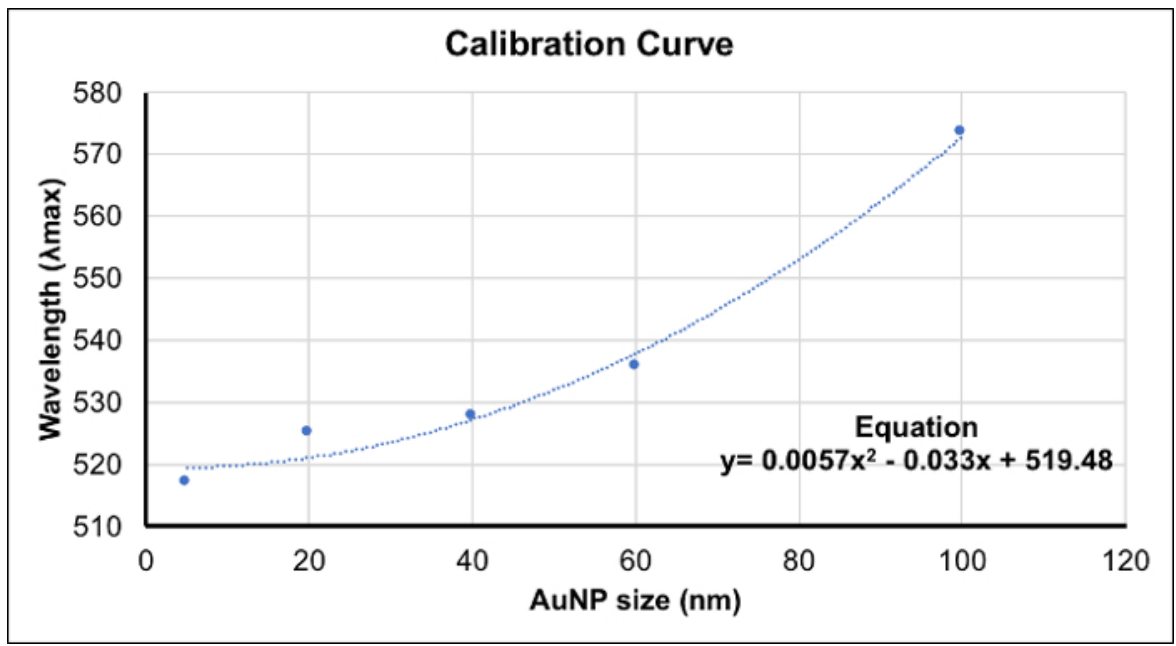

Figure 1: Calibration curve to calculate the size of the unknown sample. The plot represents the wavelengths $\left(\lambda_{\max }\right)$ and the size of the AuNPs used to plot the calibration. The plot shows only one calibration curve from one partner. Please click here to view a larger version of this figure.

\section{Representative Results}

UV-Vis is one of the most popular techniques for nanoparticle characterization as it allows the user to obtain precise analysis of properties of NMs such as Absmax and $\lambda_{\max }{ }^{6,12}$. Results of the present study represent the UV-Vis characterization of AuNP dispersions through an ILC between six participating labs.
A)

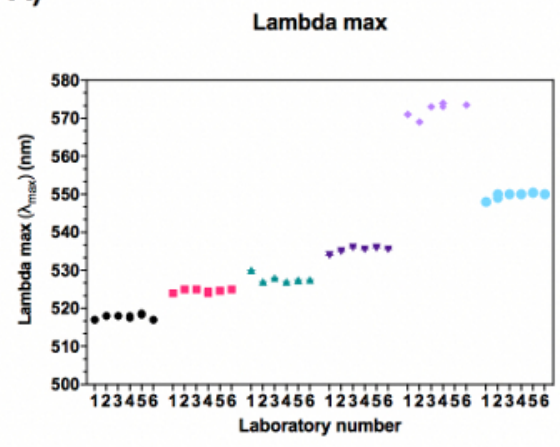

B)
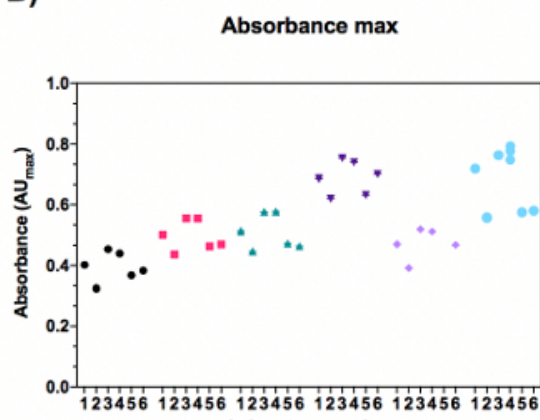
Laboratory number

- AuNP $5 \mathrm{~nm}$ - AuNP $20 \mathrm{~nm}$ - AuNP $40 \mathrm{~nm}$ V AuNP $60 \mathrm{~nm}$

- AuNP $100 \mathrm{~nm} \odot$ Unknown 
Figure 2: Lambda and absorbance results. The figures show the plots for the results reported by each laboratory for different AuNP sizes. A) Lambda max results. B) Absorbance max results. Laboratory 5 was not able to report data for 100 $\mathrm{nm}$ due to sample contamination. Please click here to view a larger version of this figure.

Results for the $\lambda_{\max }$ wavelengths showed close repeatability among the partners (Figure 2A). This was also the case for the calculated range, which was used to assess the difference between values, and which showed small differences ranging between 1.00 and $2.40\left(\lambda_{\max }\right)$ for most of the AuNP sizes (Table 1). The overall $\lambda_{\max }$ mean, calculated using the recorded mean for each laboratory for each AuNP size, similarly displayed low standard deviations for most of the sizes. The $100 \mathrm{~nm}$ size was the only exemption, as it displayed a high variation range $\left(4.66 \lambda_{\max }\right)$ between partners, leading to a greater standard deviation (572 \pm $2.00 \mathrm{~nm}$ ) compared to other AuNP sizes (Table 1). It is important to mention that laboratory 5 was not able to perform any measurements for the $100 \mathrm{~nm}$ size particles, due to contamination issues that might have compromised the repeatability of the results.

In contrast, absorbance results (Absmax) exhibited a more scattered range of data values (Figure 2B) compared to $\lambda_{\text {max }}$ results. Despite the apparently higher variability of these results between laboratories, the analysis displayed overall means with lower standard deviations and unexpected inferior variation ranges (0.11-0.21 Absmax) between laboratories compared to the $\lambda_{\max }$ results (Table 1).

\begin{tabular}{|c|c|c|c|c|c|c|}
\hline \multirow{2}{*}{ Value } & \multicolumn{6}{|c|}{ AuNP (nm) } \\
\cline { 2 - 7 } & $\mathbf{5}$ & $\mathbf{2 0}$ & $\mathbf{4 0}$ & $\mathbf{6 0}$ & $\mathbf{1 0 0}$ & Unknown \\
\hline Range $\lambda_{\max }$ & 1.45 & 1.00 & 3.00 & 2.00 & 4.66 & 2.40 \\
\hline Range Aumax & 0.12 & 0.11 & 0.13 & 0.13 & 0.12 & 0.21 \\
\hline Mean $\lambda_{\max }$ & $517.7 \pm 0.59$ & $524.6 \pm 0.45$ & $527.8 \pm 1.13$ & $535.3 \pm 0.74$ & $572 \pm 2.00$ & $549.7 \pm 0.85$ \\
\hline Mean Aumax & $0.395 \pm 0.048$ & $0.497 \pm 0.050$ & $0.509 \pm 0.057$ & $0.689 \pm 0.055$ & $0.472 \pm 0.051$ & $0.661 \pm 0.101$ \\
\hline
\end{tabular}

Table 1: Lambda and Absorbance calculated range and means. The range and overall mean and standard deviation for each AuNP size are shown. Results were calculated using the reported mean for lambda and absorbance for each laboratory (six measurements), except for the $100 \mathrm{~nm}$ size for which only 5 measurements were used to calculate the values due to a sample contamination reported by laboratory 5 .

The Z-score values were also calculated to note the distance of individual values from the overall mean. The analysis of Zscores provided information about the confidence of the ILC results, as the scores are directly related to the population distribution by displaying, in a number of standard deviations, how far a data point is from the mean ${ }^{16}$. In the results, most of the laboratories showed positive Z-score values of $0.01-1.93$ for $\lambda_{\max }$, indicating that most of the results were close to the mean and presented a normal distribution curve, as Z-scores greater than the absolute value of 2 and -2 are considered 
values that are distant from the mean and do not have a normal distribution ${ }^{16}$. The highest Z-score for Absmax was recorded for the $40 \mathrm{~nm}$ size reported by laboratory 1 , with a value of 1.93 and an $\mathrm{Abs}$ max average of $530 \pm 0$, compared to the overall mean of $527.82 \pm 1.13$ (Figure $3 \mathrm{~A}$ ). The maximum Z-score value of 1.23 for $\lambda_{\max }$ was reported by laboratory 3 along with a reported $\lambda_{\max }$ of $0.454 \pm 0$ for $5 \mathrm{~nm}$ AuNP size compared to the overall mean of $0.395 \pm 0.04$. This was followed by the $60 \mathrm{~nm}$ AuNP with a Z-score of 1.18 and an $\lambda_{\text {max }}$ mean of $0.754 \pm 0$ compared to the overall average of $0.689 \pm 0.05$. The remaining sizes displayed Z-score values from -0.04 to -1.23 (Figure 3B).
A)

Lambda max Z-scores

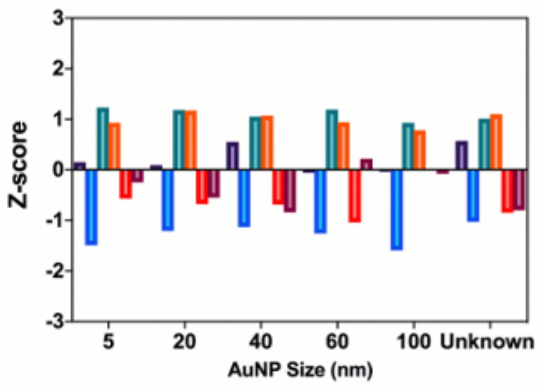

Lab 1

\section{Lab $2 \square$ Lab 3}

B)
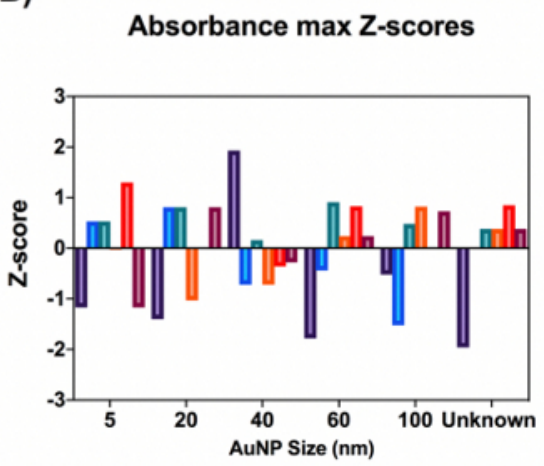

Lab $4 \square$ Lab $5 \square$ Lab 6

Figure 3: Lambda and Absorbance Z-scores. Z-scores were calculated using the results reported by each laboratory against the overall mean. A) Calculated Lambda max Z-scores. B) Calculated Absorbance max Z-scores. Please click here to view a larger version of this figure.

Results for the unknown sample showed that most of the partners calculated the size to be $76-80 \mathrm{~nm}$. The mean of laboratories $1-4$ and 6 was recorded as $78.02 \pm 1.36 \mathrm{~nm}$. Laboratory 5 reported a larger size of $109 \mathrm{~nm}$, broadening the overall average and standard deviation up to $83.18 \pm 12.70$ $\mathrm{nm}$, suggesting that this value was an outlier (Figure 4A). The Z-scores were calculated to be between -0.25 to -0.56 for all the laboratories; the only exception was for the unknown size reported by laboratory 6 , which displayed the highest positive Z-score (2.03) compared to all the measurements, which can be considered as a value that is distant from the mean (Figure 4B). 
A)

\section{Unknown nanoparticle size}

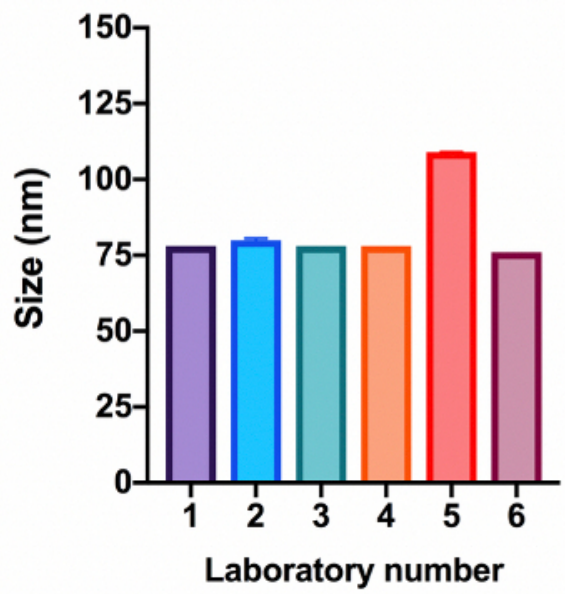

B)

\section{Z scores unknown nanoparticle size}

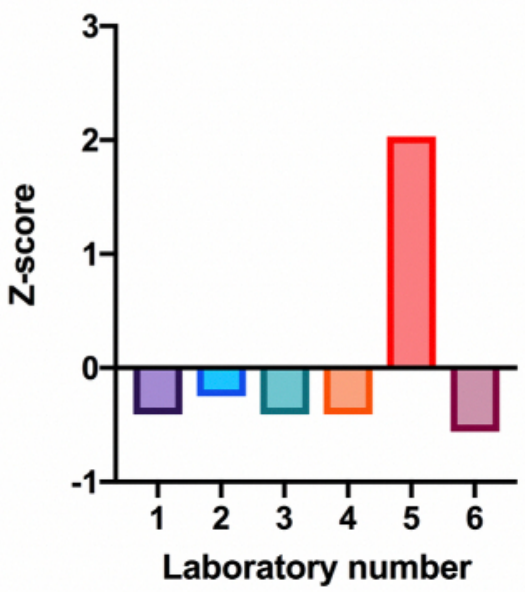

Figure 4: Unknown sample size and Z-scores. A) Reported size for each laboratory for the provided unknown sample. B) Calculated Z-scores for each individual result against the overall mean of $83.18 \pm 12.70 \mathrm{~nm}$. Please click here to view a larger version of this figure.

Supplementary Information (SI): Please click here to download this File.

\section{Discussion}

Several methods are available for the characterization of nanoscale-related properties (e.g., analytical ultracentrifugation (AUC), Scanning Electron Microscopy/ Transmission Electron Microscopy (SEM/TEM), and Dynamic Light Scattering $\left.(D L S)^{10,11}\right)$. However, these techniques lack the simplicity of UV-Vis to obtain primary results in the characterization of $\mathrm{NMs}^{12,13}$. UV-Vis is a common instrument even in not-so-well equipped laboratories, making it an unbeatable tool for the characterization of $\mathrm{NMs}^{6}$. When characterizing NMs, it is important to consider the limitations, strengths, and weaknesses of the techniques to be applied.
In the UV-Vis spectrometer, the light beam passes through the sample compartment resulting in absorption values; as a result, external vibrations, outside light, contaminants, and the user's performance may interfere with the measurement and results ${ }^{4,12}$. Similarly, when plotting a calibration curve to determine the size of an unknown sample, it is important to register all the measurements needed to construct the calibration, as missing factors may contribute to variations among measurements and users.

For example, the high variation in the overall Absmax mean of the unknown sample might be linked to differences between the laboratories due to the dependence between the beam intensity, position, and the instrument itself ${ }^{17,18}$. Furthermore, the missing data for the $100 \mathrm{~nm}$ size from laboratory 5 , due to a contamination problem, may also 
contribute to the high differences between the results, as the missing data may have affected the calibration curve and the plotted polynomial equation used to calculate the size of the unknown AuNP suspension. Certainly, reproducibility between protocols and laboratories can be complicated, as many factors might contribute to the lack of consistency in laboratory activities, resulting in researchers being occasionally unable to reproduce findings from other labs, which may lead to slower scientific progress, wasted time, money, and resources ${ }^{19}$. The successful characterization of physicochemical properties of NMs, particularly size, requires an easy-to-execute method by all participating laboratories, which can mostly be addressed by following a systematic and conceptual replication, such as the creation of an SOP, instrument training, and avoiding the use of misidentified or cross-contaminated samples ${ }^{15,19}$.

Similarly, the quality and stability of the colloid suspension are also important factors to consider, as changes in their physicochemical properties may lead to different outcomes. Therefore, to ensure their stability for longer periods, nanoparticle suspensions should be stored in the dark at $4{ }^{\circ} \mathrm{C}$. Likewise, during the shipping process, the aliquoted samples should be kept cold, as long periods at room temperature may lead to significant aggregation ${ }^{20}$. Additionally, to overcome failures in NM characterization, it is necessary to provide access to the original data, protocols, and key research materials between collaborating labs, especially, when assessing the proficiency, consistency, and reliability through an ILC ${ }^{15}$. Making these factors clear and accessible is key to achieving a successful NM characterization by any laboratory or equipment. Disregarding these aspects might result in a lack of reproducibility, accuracy, and misleading or erroneous results ${ }^{15}$. Although UV-Vis spectroscopy has been demonstrated to be the gold standard in NM characterization, it can be exploited in many other fields as it allows quantitative determination of an extended dynamic range of solutions in both inorganic and organic compounds 6,21 .

Besides, UV-Vis can be easily combined with other tools to measure a large variety of attributes, thereby improving the quality of any analysis ${ }^{22}$. Based on these features, UV-Vis is widely used in many areas such as in the biopharmaceutical field by measuring UV-Vis spectra in high concentration protein solutions, in environmental control when comparing similarities between contaminants and their product-related impurities in real time, in industrial wastewater treatments plants as part of regulations for wastewater color determination and acceptability level $^{22,23}$. Certainly, as technology progresses and more advanced features and experience become available in spectrophotometry, further broadening of the applications and parameters that can be measured using this technique will occur ${ }^{22}$. For example, in field applications, on-line UV-Vis spectrometry is a valuable tool for monitoring numerous parameters in real time and in various types of liquids, which is an exceptional feature among online sensor systems ${ }^{22}$.

The ILC described here was designed as a test of the SOP developed for UV-Vis amongst six participating labs involved in the H2020 ACEnano project. The analysis of the results demonstrated that an ILC provides valuable information to allow technical confidence in an internal method for NM characterization by each participant laboratory. Data collection in an established template confirmed consistency and faster interpretation of the results and provided a model for the estimation of the size of an unknown AuNP sample, which also displayed repeatability between results when sufficient points in the calibration curve were included. Furthermore, the results validated the 
effectiveness of UV-Vis for NM characterization as well as the importance of the creation of best practice protocols. Such an approach further provides an opportunity for the implemented procedure to contribute towards the development of a legislative framework through reproducible NM characterization protocols based on method selection and data interpretation that are relevant for accreditation regulators and research management bodies.

\section{Disclosures}

The authors declare that they have no competing interests.

\section{Acknowledgments}

ACQ would like to thank The National Council for Science and Technology (CONACyT) in Mexico for funding her PhD studies. All authors acknowledge support from the European Union Horizon 2020 Programme (H2020) under grant agreement nํ 720952, project ACEnano (call NMBP-26-2016).

\section{References}

1. Rauscher, H., Rasmussen, K., Sokull-Klüttgen, B. Regulatory aspects of nanomaterials in the EU. Chemie Ingenieur Technik. 89 (3), 224-231 (2017).

2. Hassellöv, M., Kaegi, R. in Environmental and Human Health Impacts of Nanotechnology eds J. R. Lead, E. Smith, 211-266 Blackwell Publishing Ltd. (2009).

3. Shafiq, M., Anjum, S., Hano, C., Anjum, I., Abbasi, B. H. An overview of the applications of nanomaterials and nanodevices in the food industry. Foods. 9 (2) (2020).

4. Venkatachalam, S. in Spectroscopy of Polymer Nanocomposites eds S. Thomas, D. Rouxel, D. Ponnamma, 130-157 William Andrew Publishing. (2016).
5. Bharmoria, P., Ventura, S. in Nanomaterials for healthcare, energy and environment. eds A. H. Bhat et al. 1-29, Springer, Singapore (2019).

6. Amendola, V., Meneghetti, M. Size evaluation of gold nanoparticles by UV-vis spectroscopy. The Journal of Physical Chemistry C. 113 (11), 4277-4285 (2009).

7. Yeh, Y. C., Creran, B., Rotello, V. M. Gold nanoparticles: preparation, properties, and applications in bionanotechnology. Nanoscale. 4 (6), 1871-1880 (2012).

8. European Commission. Analytical and Characterisation Excellence in nanomaterial risk assessment: $A$ tiered approach. <https://cordis.europa.eu/project/id/720952> (2019).

9. Ikhmayies, S. J. Characterization of nanomaterials. The Journal of Operations Management. 66 (1), 28-29 (2014).

10. Mourdikoudis, S., Pallares, R. M., Thanh, N. T. K. Characterization techniques for nanoparticles: comparison and complementarity upon studying nanoparticle properties. Nanoscale. $10 \quad$ (27), 12871-12934 (2018).

11. Mayeen, A., Shaji, L. K., Nair, A. K., Kalarikkal, N. in Characterization of Nanomaterials eds S. M. Bhagyaraj, O. S. Oluwafemi, N. Kalarikkal, S. Thomas, 335-364, Woodhead Publishing. (2018).

12. Tomaszewska, E. et al. Detection limits of DLS and UV-Vis spectroscopy in characterization of polydisperse nanoparticles colloids. Journal of Nanomaterials. 2013, 10 (2013).

13. Singer, A., Barakat, Z., Mohapatra, S., Mohapatra, S. S. in Nanocarriers for Drug Delivery eds S. S. Mohapatra et al. 395-419. Elsevier (2019). 
14. Perkampus, H. H. UV-VIS spectroscopy and its applications. Springer-Verlag (1992).

15. Delčev, S., Zaimovíc-Uzunovíc, N., Basíc, $H$. Participation of accredited laboratories in proficiency testing schemes and interlaboratory comparisons. Key Engineering Materials. 637 (2015).

16. Hayes, A. Financial ratios: Z-Scores values. <https:// www.investopedia.com/terms/z/zscore.asp> (2020).

17. Oliveira, O. N., Li, L., Kumar, J., Tripathy, S. K. in Photoreactive Organic Thin Films eds Zouheir Sekkat \& Wolfgang Knoll, 429-I, Academic Press. (2002).

18. Sakhno, O., Goldenberg, L. M., Wegener, M., Stumpe, J. Deep surface relief grating in azobenzene-containing materials using a low-intensity $532 \mathrm{~nm}$ laser. Optical Materials: X. 1100006 (2019).

19. ATCC. Six factors affecting reproducibility in life science research and how to handle them. <https://www.nature.com/articles/ d42473-019-00004-y\#ref-CR16> (2020).

20. Balasubramanian, S. K. et al. Characterization, purification, and stability of gold nanoparticles. Biomaterials. 31 (34), 9023-9030 (2010).

21. Łobiński, R. , Marczenko, Z. Recent advances in ultraviolet-visible spectrophotometry. Critical Reviews in Analytical Chemistry. 23 (1-2), 55-111 (1992).

22. Ojeda, C. B., Rojas, F. S. Process analytical chemistry: applications of ultraviolet/visible spectrometry in environmental analysis: an overview. Applied Spectroscopy Reviews. 44 (3), 245-265(2009).

23. Rolinger, L., Rüdt, M., Hubbuch, J. A critical review of recent trends, and a future perspective of optical spectroscopy as PAT in biopharmaceutical downstream processing. Analytical and Bioanalytical Chemistry. 412 (9), 2047-2064 (2020). 